\title{
Tessere mariniane
}

\author{
Paolo Cherchi
}

Per Arnaud Tripet

Non è esagerato dire che l'edizione dell'Adone curata da Giovanni Pozzi per i Classici Mondadori (Milano 1976) sia uno degli achievements maggiori della filologia italiana di quest'ultimo decennio. Già il fatto di aver reso accessibile un testo di difficile reperibilità è merito non trascurabile; ma il titolo maggiore in tal senso viene certo dalla cura del testo stesso. A tutti i livelli - testuale, interpretativo e storico - quest'edizione è veramente magistrale e segnerà una svolta fondamentale nella fortuna critica del Marino. Recensori impegnati come Carlo Delcorno (Lettere Italiane, XXIX, 1977, pp. 495-510) o Domenico De Robertis (L'Albero, XXVI, 1977, pp. 3-10) hanno sottolineato ripetutamente l'acume critico e la vasta informazione del Pozzi il quale non solo propone una lettura altamente stimolante - e convincente - del poema ma prodiga la sua finissima cultura classica e letteraria in genere per indicarne le fonti, per chiarirne le allusioni storiche e letterarie, per ricostruire le filigrane che operavano nella memoria dell'autore, per rendere, insomma, chiaro un testo che offre forse il più oltranzoso caso di "poesia d'arte." Non è quindi il caso di tornare su questi meriti così ovvî e singolari. L'omaggio che qui si vuol rendere a questa edizione è di tipo diverso che vorrei definire di collaborazione.

Nel corso del voluminoso commento viene espressa di tanto in tanto qualche perplessità sul significato di un vocabolo o di un'allusione; qualche volta, avendo in mente una fonte particolare, si imputa al Marino l'averla usata erroneamente, e qualche volta ci si aspetterebbe l'indicazione d'una fonte ma il commento tace. Si tratta di incidenti normali in un'edizione; ciò che è singolare, semmai, è che la loro percentuale sia estremamente bassa. Le notule - quantulaecumque - che seguono aspirano ad eliminare alcune di queste perplessità, ad indicare qualche fonte e a chiarire qualche allusione. Notule, dunque, non dettate da un occhiale da miope, ma sollecitate dallo stesso Pozzi: poiché ha lavorato a rendere solari l'officina e le pagine mariniane non gli dispiacerà di 
veder rimosso qualche atomo di polvere che inevitabilmente viene a posarsi anche sui lavori che più si avvicinano alla perfezione.

Testimonio pietoso al caso tristo fu di Sinade allora il vicin colle che d'ognintorno rosseggiar fu visto del sangue del garzon rabbioso e folle; del sangue bel che con la rupe misto tutto il sasso lasciò macchiato e molle, onde Frigia dipinti ancor ritiene

i marmi suoi di preziose vene. $(\mathrm{V}, 96)$

Il Pozzi: "Sinade: il marmo era ben noto nell'antichità . . . ma ignoriamo chi abbia potuto ispirare al M. il legame col mito di Attide."

L'ispirazione al Marino venne certamente da Stazio (Silvae, I, 5: 36-38):

sola nitet flavis Nomadum decisa metallis purpura, sola cavo Phrygiae quam Synnados antro ipse cruentavit maculis lucentibus Attis.

Negli ultimi due versi, inoltre, il Marino echeggia Claudiano (ln Eutr. II, 272-273): "picto Marmore, purpureis cui cedit Sinnada venis."

Tra i più degni germogli il panaceo le sue foglie salubri implica e mesce e '1 terebinto col dittamo ideo, da cui medico umor distilla ed esce: (VI, 128)

Il Pozzi: "sull'Ida nasce il Terebinto, secondo Plinio . . . non il dittamo, che cresce solo a Creta . . . per ragioni di versificazione e di rima il $\mathrm{M}$. non esita a combinare diversamente che nelle fonti farmaceutico-botaniche le proprietà delle piante; ed è anche questo un gioco scanzonato e allusivo." Tutto questo è probabile. Tuttavia non si può escludere che nel Marino operi un ricordo di Virgilio (Aen. XII, 411-412):

Hic Venus, indigno nati concussa dolore, dictamnum genetrix Cretae carpit ab Ida 
oppure di Stazio (Sil. I, 4: 101-102):

... Idaea profert quam Creta sub umbra

Dictamni florentis opem

o del solo Tasso (Ger. Lib. XI, 72: 5-6) che rende il testo di Virgilio:

Or qui l'angiol custode, al duol indegno mosso di lui, colse dittamo in Ida.

Fiero accidente e rapido volere,

desio che 'nchina a partorir nel bello (VI, 162)

Irresistibile, mi pare, per il primo emistichio, il rimando alla celebre canzone cavalcantiana:

Donna me prega, perch'eo voglio dire $d^{\prime}$ un accidente che sovente è fero.

Lupa vorace, ingordo mostro infame, lo cui cupo desir sempre sfavilla, che sol per satollar l'avide brame brami collo di grù, ventre di Scilla, sich'esca omai bastante a tanta fame la terra o l'acqua non produce o stilla, e dala gola tua divoratrice apena scampa l'unica fenice. (VII, 5)

Il Pozzi trova nell'ultimo verso di quest'ottava una "allusione oscura." Ma mi pare la si possa chiarire ricordando il nutrimento immateriale della Fenice indicato da Claudiano nei seguenti versi:

Non epulis saturare famen, non fontibus ullis

Adsuetus prohibere sitim: sed purior illum

Solis fervor alit, ventosaque pabula libat

Tethyos, innocui carpens alimenta vaporis. (Phoenix 13-16)

L'espressione "l'unica fenice" ricorre in rima nel sonetto "Infra gli altri difetti del libello" (V, 13) attribuito a Cino da Pistoia. Questo prelievo, come già quello della tessera precedente, portano all' osservazione generale che i riscontri coi poeti stilnovisti siano pressoché assenti in quest'edizione. 
Volea l'istoria del successo intero

Momo seguir, poiché fur colti in fallo,

e dir come di giovane guerriero

fu trasformato Alettrione in gallo,

che del duce di Tracia essendo usciero,

guernito d'armi e carco di metallo,

qual fida spia, qual sentinella accorta,

fu da lui posto a custodir la porta.

Ma perché 'l sonno il vinse e non ben tenne

per guardarsi dal sol la mente desta,

tal qual trovossi apunto, augel divenne

con lo sprone al tallon, con l'elmo in testa.

I ricchi arnesi si mutaro in penne,

il superbo cimier cangiossi in cresta,

ed or, meglio vegghiando in altro manto

accusa il suo venir sempre col canto. (VII, 225-26)

Perché non indicare la fonte della storia di Alittrone (in greco vale "gallo")? Le ottave mariniane sono traduzione quasi letterale di un passo di Luciano, Somnium seu Gallum, che cito nella traduzione latina di J. Th. Lehmann:

Gall. Audi ergo. Remotum ab omnium opinione esse, quod tibi, dico, bene novi, Micylle. Hic enim, qui nunc tibi gallus esse appareo, non ita multo ante homo eram.

Mic. Audivi quiddam tale olim sane de vobis, Gallum quendam juvenem amicum fuisse Martis, potasse cum Deo et comissatum ivisse, et amoribus illius subservisse. Quoties enim abiret ad Venerem Mars adulterii causa, adduxisse etiam Gallum: cumque Solem praesertim suspectum haberet, ne rem visam Vulcano enuntiaret, extra ad januas reliquisse semper juvenem, indicaturum sibi, quando sol appareret. Tum obdormivisse aliquando Gallum, suumque praesidium imprudentem prodidisset. Solem vero non animadversum adstitisse Veneri et re-quiesciente sine cura Marti, qui crederet indicaturum, si quis adveniret, Gallum. Atque ita Vulcanum a Sole edoctum, ipsos comprehendisse, injectis vinculis irretitos; quae olim illis parasset. Dimissum vero Martem iratum Gallo, illum ipsis cum armis in avem cognominem mutasse, ut pro galea cristam haberet in capite. Eam ob causam vos, ut excusetis vos Marti, cum nihil jam prodest, cum sentiatis orientem Solem canere diu ante, ejusque ortum significare. (Opera, graece et latine, edidit J. Th. Lehmann, t. IV, Lipsia 1855, vol. I, pp. 100-01).

Con l'ali, che sì grandi ha su le terga

vola tanto che 'l sol l'adegua apena; 
sola però l'Eternità, ch'alberga sovra le stelle, il giunge e l'incatena; la penna ancor, che dotte carte verga passa il suo volo e 'l suo furore affrena; così, chi 'l crederebbe? un fragil foglio può di chi tutto può vincer l'orgoglio. $(X, 57)$

Il Pozzi nota che la rivendicazione dell'immortalità della "penna" non ha riscontro nel contesto delle descrizioni iconologiche su cui il Marino basa la sua descrizione del Tempo. Si potrebbe però segnalare un testo di Cesare Rao: "Un vecchio alato, vestito di bianco, con un orologio in mano che figura il Tempo, fa di questo ragionamento," pubblicato nelle sue Invettive, Orationi et Discorsi (Venezia, 1597), pp. $257^{\mathrm{r}}-260^{\mathrm{r}}$. In questo ragionamento - in cui figurano molti elementi iconologici - il Tempo parla in prima persona e si difende contro coloro che credono di poterlo vincere affidando il proprio nome alla gloria delle lettere.

\section{7}

Colei ch'è prima e tiene in man le chiavi

de la sublime e spaziosa porta,

di tutte l'altre facoltà più gravi

agli anni rozzi è fondamento e scorta. $(X, 122)$

È probabile che in questa iconologia della Grammatica operi un ricordo del primo verso del Donato: "Ianua sum rudibus primam cupientem artem." Si tratta di un compendio grammaticale ricavato da Donato, che veniva chiamato "Ianua." Faceva parte degli "octo auctores" che figuravano nel curriculum ancora nel tardo Cinquecento, nonostante il ludibrio cui li avevano esposti i Rabelais o i Melantone.

E Gige v'ha che la pittura inventa ed havvi col pennello Apollodoro e Corebo è con lor, che rappresenta de la plastica industre il bel lavoro $(X, 147)$

Il Pozzi: "Gige deriva da Plinio, Nat. hist., 7, 205, ma la notizia proviene da une cattiva lettura, dovuta ad un errore di interpunzione del testo pliniano: 'athleticam Pythus, pilam lusoriam Gyges Lydus, picturam Aegyptii'. . . . Corebo è forse variante di 
Roeco, l'inventore appunto della plastica di Plinio Nat. hist., 7 , 152."

Mediatore, però, di quella cattiva lettura e della variante è Polidoro Virgilio: "Proximus est, ut post statuariam artem de picturae initiis primum dicatur. . . Picturam itaque, teste Plinio lib. 7 Gyges Lidius omnium primus invenit in Aegypto, in Graecia vero Pyrrhus Daedali cognatus, ut Aristoteli placet." (De inventoribus rerum, II, 24: "De origine picturae," Basilea 1575, p. 166). Per Corebo: "Convenit picturae contexere plasticen, hoc est, figulinam artem, quae ex terra similitudines itidem fingit. Hanc, ut Plinius in 7 ait, Chorebus Atheniensis invenit: sed idem libro 35 Dibutadi hoc assignat. .. . Sunt qui in Samo primos omnium plasticen invenisse Rhoecum et Theodorum prodant" (Ibid., lib. II, 25, p. 167: "De primis plastices inventoribus"). Basterebbero questi due riferimenti per ascrivere con certezza Polidoro Virgilio al novero degli auctores del Marino. La nozione relativa a Corebo "di Atene [che] ritrovò il far i vasi della terra" appare anche nel Vago e dilettevole giardino di Luigi Contarino nella sezione dedicata agli "inventori di tutte le scienze ed arti" (p. 409 dell'ed. di Vicenza, 1602).

Contro la luna il fier quadrato giunge, laqual dinotatrice è dela morte e per direzion le si congiunge minacciandoti pur l'istessa sorte, perché, com'anaretico, l'aggiunge virtù nel mal più vigorosa e forte; e l'un e l'altro in loco tal s'annida che ne divien nocente ed omicida. (XI, 181)

Il punto óscuro di quest'ottava, che fa parte dell'oroscopo di Adone, è la parola anaretico. La Colombo (Cultura e tradizione nell'Adone di. G. B. Marino, Padova 1967) trova la voce (sostantivo maschile) di "significato oscuro" e non attestato altrove. "Non registrata" la dice il Pozzi. Una proposta di spiegazione viene da Carlo Delcorno (art. cit. p. 507) che su indicazione del Du Cange si orienta verso la farmacopea: "farmaco che potenzia le energie." Ma il senso non ne risulta chiarito. Bisognerà invece attenersi al linguaggio dell' astrologia, e qui si troverà la spiegazione.

Anaretico è aggettivo formato su anareta. Questa voce rende il sostantivo greco ' $\alpha \nu \alpha \iota$ Łetés che significa "distruggitore" o occisor secondo K. Gesner, Lexicon Graecolatinum (Basilea 1545 a.v.). Il termine figura in un contesto astrologico nel terzo libro, cap. 14 
della Paraphrasis in Ptolomei libros IV di Proclo, e nella traduzione latina di Leone Allacci (Ludguni Batavorum, 1654, p. 190) viene reso come pianeta interfector. Purtroppo non mi è possibile indicare alcun testo latino che adotti e adatti la voce greca. Ma che questa sia la pista giusta è confermato indirettamente da un passo inglesi di William Lilly:

I have delivered what the Ancients wrote of Hyleg, but as yet I rest not satisfied either how to take the Hyleg aright, or whom most properly to call the Killing, Interficient or Destroying Planet, or more artificially Interfector or Anareta; yet the Ancients with great reason have delivered that the Anareta or Interficient Planet, is he who is placed in the eighth house, either five degrees before the cups of the house, or 25 degrees after. (Christian Astrology modestly treated in three books, Londra, 1647, cap. CIV, p. 529-530).

Il termine anareta è registrato con questo significato in vari dizionari d'astrologia fra cui ricorderò soltanto quello di James Wilson, (Boston 1885) e di H.E. Wadeck (Secaucus, N.J. 1973); ed è registrato anche dall'Oxford English Dictionary che rimanda al passo di W. Lilly qui citato. L'OED registra anche l'aggettivo anaretic e anaretical. ${ }^{1}$

De' popoli che domi avea con l'armi

la pompa trionfal traea quel giorno

e da' vinti Geloni e da' Biarmi

al suo tracio terren facea ritorno. (XII, 41)

Protagonista di questo trionfo è Marte che sta per esser visitato da Gelosia. Fra i popoli vinti, i Biarmi non sono stati identificati. Ma basta aprire la Historia Gentium Septentrionalium di Olao Magno al primo capitolo per vedere che si tratta di una popolazione dell'estremo nord dell'attuale Finlandia, di una regione, cioè, che si chiama Biarma:

Biarma igitur est regio Septentrionalis, cuius zenit est in ipso polo Arctico, eiusque Horizon est idem cum circulo aequinoctiali: qui Zodiacum in duas partes aequales intersecans, efficit, ut una medietas totius anni sit unus dies artificialis, et altera medietas una nox: et ita totus annus esset ibi unus dies naturalis. ... (ed. Basilea, 1567, p. 1)

La popolazione viene chiamata alternativamente Biarmi o Biarmantes. Purtroppo non ho avuto accesso alle varie traduzioni ita- 
liane di quest'opera che forse il Marino consultò; ma esse renderanno sicuramente il testo latino con Biarmi o Biarmanti.

Come con sua virtù sottile e lenta ch'ha vigor di velen, rigor di ghiaccio, s'al'esca la torpedine s'aventa toccando l'amo e penetrando il laccio, scorre ratto ala canna ed addormenta del pescator assiderato il braccio e, mentre per le vene al cor trapassa, tutto immobile e freddo il corpo lassa, (XII, 61)

Nessuna indicazione sulla fonte di questa proprietà della torpedine già indicata da Plinio: "torpedo ... etiam procul et e longiquo, vel si hasta virgave attingatur, quamvis praevalidos lacerto torpescere, quamlibet ad cursum veloces alligari pedes" (Nat. Hist., XXXII, 7). La fonte diretta - se non interviene qualche mediazione in volgare - è quasi certamente l'idillio che Claudiano dedica alla torpedine. Qui si trova l'immagine della canna e della lenza conduttrice del veleno della torpedine:

Si quando vestita cibis incautior aera

Hauserit, et curvis fraenari senserit hamis;

Non fugit, aut vano conatur vellere morsu:

Sed propius nigrae jungit se callidae setae,

Et meminit captiva sui, longeque per undas

Pigra venenatis effundit flamina venis.

Per setam vis alta meat, fluctusque relinquit

Absentem tentura virum. Metuendus $a b$ imis

Emicat horror aquis, et pendula fila secutus

Transit arundineos arcano frigore nodos,

Victricemque ligat concreto sanguine dextram. (vv. 12-22)

\section{2}

Saetta è la beltà che l'alma uccide subitamente e passa al cor per gli occhi.

Fu la beltà ch'ella in mal punto vide apunto come folgore che scocchi.

Fu l'occhio che seguì scorte mal fide qual ghiaccio fin, s'avvien che raggio il tocchi, ch'arid'esca vicina accender suole e ferir di scintille il viso al sole. (XII, 178) 
Il Pozzi: "qual ghiaccio fin: non sappiamo indicare l'origine di questo mostro di natura."

Per arrivare ad una spiegazione del testo bisogna vedere nel "ghiaccio fin" una lente, e quest'equivalenza ci porta ad un topos caro ai trovatori e ai poeti nostrani delle origini. La sua fonte scientifica è in Marbodo il quale sviluppa una nozione già presente in Seneca (Nat. Quaest. III, 25, 12) e in Plinio (Nat. Hist. XXXVII, 23) e ripresa da Claudiano in vari dei suoi epigrammi. Secondo tale nozione la neve delle Alpi compressa dagli anni si trasforma in ghiaccio, e il ghiaccio, sotto ulteriore pressione si trasforma in cristallo. Ecco i versi del De lapidibus di Marbodo giusta la recente edizione di John M. Riddle (Sudhoffs Archiv., Zeitschrift für Wissenschaftgeschichte, 1977, che sostituisce quella della P.L. 171):

Cristallus glacies multos durata per annos,

Ut placuit doctis, qui sic scripsere, quibusdam,

Germinis antiqui frigus tenet atque calorem

Sed certum cunctis, nec stat dubitabile cuiquam

Quod lapis hic soli subjectus concipit ignem,

Admotosque sibi solet hinc accendere fungos. (550-58)

Quest'immagine di sapore adynatico piacque a Peire Vidal ("de la freja nieu Nais lo cristals, don hom trai fuec arden," ed. Avalle, XXXVIII, 30-31 con ottimo commento) e ai poeti siciliani: basterà ricordare la prima quartina del sonetto di Giacomo da Lentini:

A l'aire claro ho visto ploggia dare,

ed a lo scuro rendere calore;

e foco arzente ghiaccia diventare

e freda neve rendere calore. (ed. Contini, Poeti del Duecento, I, p. 78)

A nessuno sfuggirà l'ardita transumptio che il Marino ricava da questo vecchio topos. Il freddo cristallino ("il cristallo, onde sol procede il lume" VI, 33) di Falsirena è la lente che accende il suo cuore.

Or colta ha l'erba rara e vigorosa, non so ben dire in quale estrania terra, contro la cui virtù meravigliosa con mille chiavi indarno uscio si serra, e se le piante alcun destrier vi posa ne svelle i chiodi e lo discalza e sferra. (XIII, 127) 
Quest'erba che la colomba porta ad Adone imprigionato ha un nome: "erba luccia." Così la chiama l'autore del Mare Amoroso:

E potesse avere de l'erba luccia, che sa sfermare ciascuna fermatura. (ed. Vuolo, 237-38)

Il Vuolo indica vari testi medievali in cui appare quest'erba dalle proprietà magiche; ma il Mare Amoroso è l'unico luogo in cui questo nome appaia. In un intervento successivo (Rivista di Cultura Classica e Medievale, VII, 1965 [studi in onore di A. Schiaffini], p. 1160) il Vuolo indica la presenza dell'erba e delle sue proprietà in Sardegna dove vien chiamata erva luttsa (cfr. A. Sanna, "Note Sardo-logudoresi," in Studi sardi, XII-XIII, 1952-53, p. 24). Si tratta di una leggenda molto diffusa. Ne trovo un campione in Ispagna, dove l'erba è chiamata "pito":

Pito, un pajaro fue elque dio noticia dela yerva llamada de su Nombre

Pito, cuya virtud estal que abre

los cerrados conductos, quiebra el hierro,

cual vemos en Sevilla, a donde nace

esta yerva del Pito, sinque sea

vista, ni conocida de ninguno

mas de que la esperiencia loa enseñado.

Questo passo (ricavato da Juan de la Cueva, Los inventores de las Cosas. A Critical Edition and Study, a cura di B. Weiss e L. C. Pérez, University Park, Londra, 1980, libro III, 280-87) e altri che si potrebbero ricordare (tanto per stare in area iberica, si pensi alla Gaviota di Fernàn Caballero, cap. IX), provano quanto fosse diffusa questa leggenda: il che rende pressoché irrilevante l'indicazione precisa della fonte del Marino; a meno che non si voglia apprezzare ancora una volta il suo modo di "riscrittura," e vedere, per esempio, se la proprietà di scalzare o di sferrare un "destriero" non sia una sua spiritosa invenzione.

e 'l fino specchio di diamante terso che risplendea nel pettoral d'Astreo, in cui sovente il popolo converso ogni evento augurava o buono o reo e qualor fosco o pur di sangue asperso rendea 'l color, secondo l'uso ebreo, temea di morte o danno altro futuro, videsi lampeggiar lucido e puro. (XVI, 60) 
Il Pozzi, riferendosi all'uso ebreo, chiosa: "difficile dire a che cosa alluda; non ha certo riscontro nelle vesti sacerdotali descritte dalla Bibbia nel Levitico e nei Numeri." Ma non mi pare dubbio che il Marino alluda qui all'Urim e Thummim che la Vulgata rende con doctrina et veritas. La descrizione più ampia di questo mezzo per consultare il volere divino si ha nell'Exodus, 28, 15-30. Si tratta di una borsa o pettorale in cui è contenuto un gioiello fatto di varie pietre preziose di vario colore e forma. Solo il sommo sacerdote poteva usare questo "pettorale del giudizio" ("rationale iudicii"), come viene chiamato, per conoscere il volere di Dio nei momenti di grande pericolo per l'intero popolo ebraico. Ora, le descrizioni di questo pettorale sono alquanto diverse da libro a libro; ma ciò che rimane sempre oscuro è il modo in cui vien data la risposta: si dice (I Sam. 14, 41) come si deve porre la domanda, ma non come Dio risponderà. Ma un testo frequentato dal Marino (cfr. Dicerie sacre, ed. Pozzi, passim.) ci descrive come ciò avvenisse: proprio attraverso il riflesso della luce. L'autore è Giuseppe Flavio e il testo sono le Antiquitates Judaicae che cito nella traduzione latina di G. Dindorfius:

Verumtamen quod de pontificis vestitu praetermisi, persequi volo: nam is omnem prophetis fraudum et praestigiarum ansam praecidebat. Verum si qui eo devenirint ut Dei auctoritate abutantur, Deo quidem liberum esse [reliquit] quando sacris interesse velit vel non adesse: id quod non Hebraeis solum manifestum esse voluit, sed et alienigenis qui his forte intervenirent. Nam ex lapidibus quos super humerum pontificem ferre praedixi (erant autem sardonyches, atque eorum naturam ostendere supervacuum puto, quum a nemine ignorentur,) contigit ut micaret, quoties Deus sacrificiis adesset, alter qui in dextro humero fibulae vice portabatur, fulgore inde exiliente, ut etiam procul positis illusceret, quum prius lapis eo careret splendore. Et certe hoc ipsum admirationem meretur apud omnes, qui non ex contemptu religionis sapientiae opinionem aucupantur. Sed quod hoc etiam mirabilius est jam dicturus sum. Nam per duodecim lapides, quos in pectore pontifex essenae adsutos gestat, Deus victoriam pugnaturis praenunciabat. Tantus enim ex iis effulgebat splendor, quum nondum exercitus moveretur, ut toti populo manifestum fuerit ipsis auxilio adesse Deum. (lib. III, $8-$ in Opera, graece et latine, recognovit G. Dindorfius, Parigi, 1855, pp. 100-01)

Tal cervo a cui talor tronca o caduta

la selva sia dele ramose corna, vergognosetto in solitaria e muta

valle s'appiatta e 'n tana erma soggiorna. (XVI, 88) 
Non sarebbe stato inopportuno indicare almeno la fonte remota di questa nozione. È ancora una volta Plinio che dei cervi ci dice: "Cornua mares habent, solique animalium omnibus annis stato veris tempore amittunt. Ideo sub ista die quam maxime invia petunt; latent amissis velut inermes, sed et hi bono suo invidentes." (Nat. Hist., VIII, 115)

Un frontal d'etiopico ametisto

l'adusta fronte illuminando inaura,

e di qualunque cor languido e tristo

la mestizia rallegra, il duol restaura; (XVI, 113)

Il Pozzi non riscontra queste proprietà rasserenanti nei canonici testi di Plinio e di Marbodo. Ma tali proprietà non sono sconosciute ai lapidari medievali. Bartolomeo Anglico, per es., parlando dell' ametisto dice: "virtus eius contra ebrietatem est, et facit vigilem, et malam repellit cogitationem, et bonum confert intellectum" (De rerum proprietatibus, XVII, 10, ed. di Francoforte, 1601, p. 724). Tuttavia - e questa è la spiegazione più cogente bisogna ricordare che un tipo di ametisto "descendit ad hyacinthum" (ibid., p. 723), ed è probabile che il Marino abbia scambiato la proprietà delle due pietre. Del giacinto, che si trova anch'esso in Etiopia, B. Anglico scrive infatti "est virtus singularis, secundum auctores, quod confert laetitiam et contrariatur melancholicae qualitati" (XVI, 54, p. 743). E fra gli autori bisognerà ricordare, appunto, Marbodo:

Jacinti species docti tres esse loquuntur;

nam sunt granati, sunt citrini, venetique.

Confortativae cuncti virtutis habentur,

tristitiamque fugant et vanas suspiciones. (ed. cit., vv. 214-17)

\section{7}

Prima che dale man celesti e sante fusse in colmo fornita opra si bella, nove volte lucifero in levante precorse al gran camin l'alba novella e mutato destriero anco altrettante guidò notturna la più bassa stella. Comparso il nono sol, comparve in tutto l'edificio superbo apien costrutto. (XIX, 347) 
Riprende Stazio:

Roscida iam novies caelo dimiserat astra Lucifer et totidem Lunae praevenerat ignes mutato nocturnus equo, nec conscia fallit sidera et alterno deprenditur unus in ortu; mirum, opus adcelerasse manus: stat saxea moles (238-42)

Questi versi sono ricavati dal canto sesto della Tebaide, canto che la "ronchetta" mariniana ha potato ripetutamente e molto più sistematicamente di quanto i riscontri del Pozzi non facciano capire. È il canto che Stazio dedica al funerale di Ofelte e ai giochi funebri in suo onore, e il Marino lo echeggia negli ultimi due canti del suo poema. Ecco alcuni riscontri che si possono aggiungere a quelli del Pozzi. Il taglio del bosco per allestire la pira è così sistematico che Stazio osserva: "Dat gemitum tellus" (107) e il Marino "Geme la terra" (XIX, 355) e continua utilizzando altri materiali di Stazio. Un altro riscontro: "signum luctus cornu grave mugit adunco Tibia" (120-21); e il Marino

Ed ecco il rame già curvo, forato con lugubre muggito alto risona e che 'ncominci l'ordine schierato de l'essequie a partirsi il segno dona; (XIX, 359)

Alcuni particolari del rogo hanno il loro corrispondente in Stazio:

Più nobil fiamma in terra unqua non arse, né cener mai più ricco si compose. Chi di candido latte urne vi sparse e chi di negro vin tazze spumose. Altri le mani ancor non avea scarse di biondo mele e di più rare cose. Altri del sangue degli uccisi armenti abbeverava le faville ardenti. (XIX, 400)

I primi due versi rendono "Ditantur flammae; non umquam opulentior illic Ante cinis" (206-07); i rimanenti amplificano e adattano "mella Spumantesque mero paterae verguntur et atri Sanguinis et rapti gratissima cymbia lactis" (210-12). Sulla falsariga di Stazio (224-26) è costruita buona parte dell'ottava 401 che descrive gli oggetti che vengono gettati nel rogo. Più cogenti - perché più precisi - sono i riscontri dell'ottava 402:

Indi il bel rogo ancor, secondo rito, prende da manca a circondar tre volte, 
ed inchinando il busto incenerito

le bellezze saluta in aria sciolte.

Ma poiché già Vulcan langue sopito

e l'ossa amate ha in polvere rivolte,

di propria mano il cenere rimaso

raccoglie e serra entro 'l marmoreo vaso.

Nei primi quattro versi vien reso: “. . . versis ducunt insignibus ipsi Graiugenae reges, lustrantque ex more sinistro Orbe rogum et stantes inclinant pulvere flammas. Ter curvos egere sinus . .." (214-17). Negli altri quattro vien ripresa l'immagine del languente Vulcano: "Finis erat; lassusque putres iam Mulciber ibat In cineres" (234-35).

e tutti neri gli abiti costrusse, i cigni istessi neri ebber le penne, le bianche penne co' purpurei rostri tutte eran tinte de' più puri inchiostri. (XIX, 367)

Propriamente un adynaton realizzato che ha come base il verso di Giovenale (VI, 165):

rara avis in terris nigroque simillima cycno.

Ma qui non siamo in terra. Siamo a Cipro, un mondo perfetto, e per così dire, alla rovescia!

Sovra un bel soglio d'or preme Lieo

la fera ch'idolatra è dela luna. (XIX, 381)

Il Pozzi: "Passo di difficile spiegazione. Forse [la fiera] è la pantera di cui si diceva che avesse sul dorso una macchia che cresceva e diminuiva secondo le fasi lunari." Proporrei, invece, di identificare la "fera" con l'elefante fra le cui attitudini quasi umane Plinio ricorda anche la "religio quoque siderum solisque ac lunae veneratio. Auctores sunt in Mauretaniae saltibus ad quendam amnem cui nomen est Amilio nitescente luna nova greges eorum descendere ibique se purificantes sollemniter aqua circumspergi atque ita salutato sidere in silvas reverti vitulorum fatigatos prae se ferentes" (Nat. Hist., VIII, 2). E l'elefante è certamente "fera" più atta a reggere una sedia gestatoria. 
Chiama questo suo gioco empio e profano saravanda e ciaccona il novo ispano. $(X X, 84)$

Chiosa: "'novo ispano': l'americano? Non troviamo riscontro di questa opinione sull'origine del ballo." Dietro quest'affermazione c'è sicuramente l'autorità della Colombo (op. cit., p. 114 sg.): "La ciaccona ebbe origine in Spagna, ove è menzionata a partire dal 1591." La Colombo rimanda al Grove, Dictionary of Music nell'edizione del 1954 alla voce chaconne. Ma il New Grove Dictionary, ed. 1980, non lascia dubbi sull'origine della danza: "The earliest literary reference to chacona occurs in a poem by Mateo Rosas de Oquendo, describing events in Peru in 1598. Its New World origin is mentioned by numerous Spanish writers such as Cervantes and Lope de Vega who frequently linked it with the zarabanda." Del resto, per una rassegna di testimonianze in questo senso e per la bibliografia sull'argomento, sarebbe stato sufficiente consultare A. Machabey, "Les origines de la chaconne et de la passacaille," in Revue de Musicologie, I (1946), pp. 1-21.

carta è il ciel, l'ombra inchiostro e penna il raggio. (XX, 248:5)

Forse in filigrana c'è il topos "Wenn der Himmel wär Papier" illustrato da E.R. Curtius, Europäische Literatur und lateinisches Mittelalter (Berna, 1948), p. 318.

Nell'episodio di Ciparisso narrato ad Adone da Mercurio (V, 49-64) si ha la descrizione del cervo amato dal giovinetto; e qui i seguenti versi:

D'oro l'orecchie e d'or la fronte adorna,

gli circonda la gola aureo monile

ch'un tal breve contien: "ninfe e pastori,

di Diana son io, ciascun m'onori." $(\mathrm{V}, 51,5-8)$

Nel modello Ovidiano (Metam. X, 106-42) manca l'iscrizione nel collare. Marino l'ha probabilmente costruita avendo in mente la 
leggenda della cerva di Cesare tramandata col motto iscritto nel collare "Noli me tangere, Caesaris sum." Per la fortuna di questo monito - da Solino all'emblematica cinque-seicentesca basata in parte su Petrarca (RVF, CXC) - si veda lo studio di M. Bath, "The Legend of Caesar's Deer," in Medievalia et Humanistica, IX (1979), 53-66, con ampia bibliografia.

Ben conosco il mio fallo e men'aveggio, ma qual egro assetato, amo il mio peggio. (XII, 219, 7-8)

In queste parole di Falsirena opera ancora una volta la tecnica conflativa mariniana. Il primo emistichio del verso 7 è ricalcato sul petrarchesco "Ch'i' conosco 'l mio fallo e non lo scuso" (RVF, CCCLXIV, 14). Il primo emistichio del verso successivo è certamente ricavato da Ovidio: "Nitimur in vetitum semper cupimusque negata; Sic interdictis inminet aeger aquis" (Amores, III, 4, 17-18). Il secondo emistichio, quindi, echeggia nuovamente il Petrarca: "E veggio '1 meglio et al peggior m'appiglio" (RVF, CCLXIV, 136) dove si esprime un concetto identico a quello fermato nel verso sopra riportato ma presentando un ordine inverso dei termini che costituiscono l'alternativa. E dietro quest' ultimo verso della canzone petrarchesca c'è il modello ovidiano: "Video meliora proboque, Deteriora sequor" (Metam. VII, 20-21).

- E tu chi sei? (replica l'altro) e donde

il primo a cercar brighe esci fra tanti?

Spesso quand'altri per timor s'asconde,

chi di tutti è il peggior si tragge avanti. -

- Son chi mi sono, e qual mi sia (risponde)

son più di te, che sì ti stimi e vanti

e di qualunque al par di te s'apprezza

degno di posseder quella bellezza. - (XX, 201)

Protagonisti di queste ritorsioni sono l'arrogante Esperio, spagnolo, e l'indignato Bardo, toscano. Pare di assistere ad una scena di commedia di cappa e spada spagnolesca. È curioso, infatti, osservare che il "son chi mi sono" di Bardo corrisponda ad un fortunatissimo cliché del teatro spagnolo del siglo de oro: "soy quien soy," calco del biblico "sum qui sum," è una delle espressioni più frequenti della honra nobiliare spagnola (si vedano gli studi di L. 
Spitzer "Die 'Estrella de Sevilla' und Claramonte," in ZfrPh. LIV, 1934, 563, e "Soy quien soy," in Nueva Revista de Filología Hispánica, I, 1947, 113-27). Mi chiedo se il Marino non intendesse satirizzare l'arroganza spagnola mettendo questo topos in bocca d'un toscano che, si direbbe, risponde così per le rime ad uno spagnolo. Che il "son chi sono" suonasse pretenzioso e ridicolo ce lo potrebbe confermare il goldoniano Marchese di Forlimpopoli, il nobile spiantato che può sempre supplire a tale miseria un tronfio "son chi sono." Tuttavia col Goldoni siamo in clima diverso e forse il "son chi sono" si era cristallizzato, oltre che nelle commedie spagnolesche, anche nei lazzi della commedia dell' arte.

\section{University of Chicago}

\section{NOTE}

1 Avverto il lettore di una mia svista che spererei venga giudicata veniale: il termine "anaretico" qui discusso era stato già trattato da Gianfranco Folena (Lingua nostra, XXXVIII, 1977, 31) con risultati simili a quelli da me raggiunti, ma con dati bibliografici in parte diversi: le due note si potrebbero integrare. 